\title{
Optical Code-Division Multiplexing (OCDM) Networks Adopting Code-Shift Keying/Overlapping PPM Signaling: Proposal and Performance Analysis
}

\author{
Ahmed E. Farghal, Hossam M. H. Shalaby, Senior Member, IEEE, \\ Kazutoshi Kato, Senior Member, IEEE, and Ramesh K. Pokharel, Member, IEEE
}

\begin{abstract}
Optical code-division multiplexing (OCDM) systems use short pulses compared with bit-duration to achieve high transmission rate. The use of short pulses poses several problems as a result of group velocity dispersion (GVD), intersymbol interference (ISI) (due to avalanche photodiode (APD) buildup time), and receivers limited bandwidth. In this paper, an OCDM system employing code-shift keying (CSK) and overlapping pulseposition modulation (OPPM) signaling is proposed and theoretically investigated. By using CSK while maintaining same data rate, the chip duration can be increased to counteract the GVD effect in 2D OCDM systems. Moreover, by increasing the chip duration, the chip rate is decreased and the stringent requirement on receiver bandwidth is relaxed. In addition, using overlapping property in OPPM allows for further chip duration increase. We consider using correlation receivers with hard-limiters and APDs at the receiver side. The bit error probability (BEP) of the proposed system is derived taking into account the impacts of APD noise, thermal noise, GVD, ISI, and multiple-access interference (MAI). A performance comparison between OOK-, PPM-, OPPM-OCDM and the proposed system is carried out. Our results reveal that the use of CSK/OPPM-OCDM with data rate constraint allows the reduction of MAI, GVD and ISI effects with improved spectral efficiency.
\end{abstract}

Index Terms-Optical code division multiplexing (OCDM), APD noise, intersymbol interference (ISI), code-shift keying (CSK), overlapping pulse-position modulation (OPPM).

\section{INTRODUCTION}

$\mathbf{O}$ PTICAL code division multiplexing (OCDM) is a very promising candidate for all-optical local and metropolitan area networks, as it enables fully asynchronous transmis-

Manuscript received February 19, 2015; revised May 27, 2015, July 22, 2015, and August 1, 2015; accepted August 4, 2015. Date of publication August 12, 2015; date of current version October 15, 2015. This work was supported by the Egyptian Ministry of Higher Education. The associate editor coordinating the review of this paper and approving it for publication was W. C. Kwong.

A. E. Farghal is with the Department of Electronics and Communications Engineering, Egypt-Japan University of Science and Technology (E-JUST), Alexandria 21934, Egypt (e-mail: ahmed.farghal@ejust.edu.eg).

H. M. H. Shalaby is with the Department of Electronics and Communications Engineering, Egypt-Japan University of Science and Technology (E-JUST), Alexandria 21934, Egypt, on leave from the Electrical Engineering Department, Alexandria University, Alexandria 21544, Egypt (e-mail: shalaby@ieee.org).

K. Kato and R. K. Pokharel are with the Graduate School of Information Science and Electrical Engineering, Kyushu University, Fukuoka 819-0395, Japan (e-mail: kato@ed.kyushu-u.ac.jp; pokharel@ed.kyushu-u.ac.jp).

Color versions of one or more of the figures in this paper are available online at http://ieeexplore.ieee.org.

Digital Object Identifier 10.1109/TCOMM.2015.2467372 sion, low access delay, potentially secure data transmission, support for bursty traffic, and soft-capacity on-demand.

Two-dimensional (2D) wavelength-hopping time-spreading (WHTS) OCDM codes, where encoding is performed in both time and wavelength domains, can support larger code cardinality using much shorter code lengths than one-dimensional (1D) time spreading codes under same bit-rate constraint [1]-[5]. This is particularly useful in high bit-rate OCDM applications, where the number of time slots is restricted.

Avalanche photodiodes (APDs) are commonly used in many high-speed optical receivers due to their internal optoelectronic gain [6]. The three main competing factors that govern APDbased optical receivers performance at high speed are: (i) the avalanche noise of the APD; (ii) the buildup time which limits APD speed by inducing intersymbol interference (ISI); and (iii) the APD dark current [7].

Group velocity dispersion (GVD) is one of the physical impairments that limits the performance of 2D OCDM systems [8], [9]. Due to the differing group velocities of a codeword's wavelength components, GVD causes temporal spreading of chips, which results in chip peak-power reduction. Moreover, in TS OCDM systems the chip rate is very large ( $\gg$ Gchip/s) and ISI resulting from the APD's buildup time cannot be neglected.

Code-shift keying (CSK)-OCDM, where different sequences of $\log _{2} M_{c}$ bits of a message are encoded into different $M_{c}$ codewords, has been demonstrated to increase the data rate, increase the spectral efficiency, enhance the OCDM network confidentiality, and relax the effects of GVD [10]-[13]. However, CSK requires codes with very large cardinality. In addition, compared with on-off keying (OOK)-OCDM, CSK would produce significantly more multiple-access interference (MAI) for a given number of simultaneous active users as a result of data bit " 0 " is being transmitted also [10].

One possible solution to reduce MAI is the use of pulseposition modulation (PPM)-OCDM as transmission occurs only in a small fraction of the frame, thus reducing the probability of chip interference [14]. However, in PPM techniques, the laser pulsewidth must be shortened to be able to achieve the requirements on the throughput while using very long code sequences. Thus, PPM is more susceptible to GVD and ISI. In [15] the authors combine multi-code modulation (MCM) and PPM in a new modulation scheme, namely multi-code PPM (MCPPM) to simultaneously mitigate the impact of both GVD and MAI. In overlapping PPM (OPPM) techniques, where 


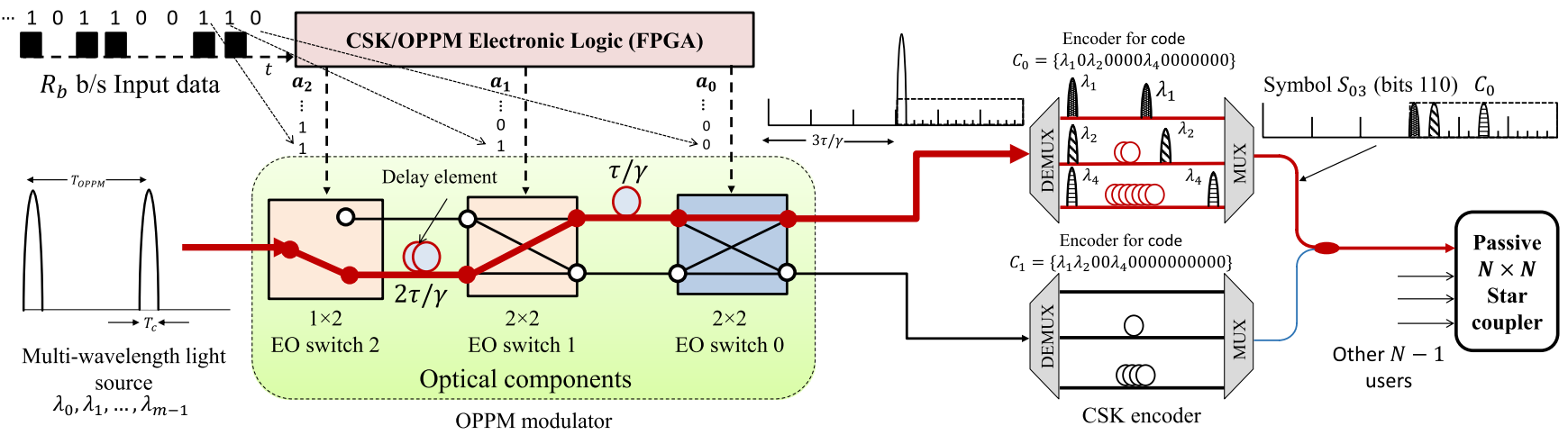

Fig. 1. CSK/OPPM-OCDM encoder for $M_{c}=2, M_{p}=4, \gamma=3, L=15$, and $w=3$.

overlapping between neighboring slots is allowable, it is possible to offer higher throughput without the need to decrease the laser pulsewidth [16], [17].

In the present paper, we propose and analyze an alternative technique that combines both CSK and OPPM-OCDM techniques; namely CSK/OPPM-OCDM. In this work, we only consider unwrapped OPPM signaling scheme [17]. The idea is that, in OCDM, at specified bit error probability (BEP) level, there is always a set of unused codes that can be exploited by assigning a set of $M_{c}$ codewords to each user. 2D onecoincidence frequency-hopping code/optical orthogonal code (OCFHC/OOC) codewords are used as the signature sequences in the proposed OCDM system [5]. In order to increase the discrimination between the desired codeword and interfering codewords in addition to minimizing MAI between active users, the auto- and cross-correlation are bounded by one. Under the condition of the same bit rate, employing CSK- or CSK/OPPMOCDM permits increase of the chip duration. Thus, the effects of GVD are greatly reduced. In addition, as the chip duration increases, the chip rate decreases, relaxing the receiver bandwidth constraints and reducing the ISI effect.

The remainder of the paper is organized as follows. The system model of CSK/OPPM-OCDM is described in Section II. In Section III, an upper bound on BEP is derived taking into account the effects of MAI and GVD. In addition, the analysis includes the effects of APD noise, dark current, thermal noise, and ISI. Next, we numerically compare the BEP of the proposed system with that of OOK-, PPM-, and OPPM-OCDM systems in Section IV under the peak transmitted power constraint. Finally, conclusions are drawn in Section V.

\section{PRoposed CSK/OPPM-OCDM SCHEME}

In this section we describe the transmitter and receiver architectures of our network, which is composed of $N$ users.

\section{A. Transmitter Side}

The transmitter of each user $k \in\{1,2, \ldots, N\}$ is composed of one OPPM modulator and $M_{c}$ 2D OCDM encoders (or one tunable). OPPM modulator only involves time delaying of the optical pulses into one of the $M_{p}$ overlapping spreading intervals (called slots) that constitute an OPPM frame [17],
[18]. In OPPM technique, a slot of duration $\tau=L T_{c}$, where $L$ is the code length and $T_{C}$ is the chip duration, is divided into $\gamma \in\left\{1,2, \ldots, M_{p}-1\right\}$ subslots of width $\tau / \gamma$ each, where $\gamma$ is the overlapping index. Each slot is forced to overlap with its adjacent slots so that there is an overlap of $(\gamma-1) \tau / \gamma$ between any two adjacent slots. An optical pulse coming from a multiwavelength light source of width $T_{c}$, repetition rate $1 / T_{O P P M}$, and peak power $w P_{c}$ is time delayed depending on the incoming data, where $T_{O P P M}=\left(M_{p}-1+\gamma\right) L T_{c} / \gamma$ is the full width of OPPM time frame, $P_{c}$ is the transmitted peak power per chip, and $w$ is the code weight. The CSK/OPPM electronic logic unit is merely a serial-to-parallel $(\mathrm{S} / \mathrm{P})$ converter where a serial data bit stream $\left\{d_{0}, d_{1}, d_{2}, \ldots\right\}, d_{i} \in\{0,1\}$, is segmented every $\log _{2} M$ bits, where $M=M_{c} \times M_{p}$. The segmented bits are then parallized to generate $a_{i}, i \in\{0,1, \ldots, M-1\}$ trigger signals that will employed for configuring the electrooptic (EO) switches as shown in Fig. 1. The S/P converters can be implemented using low cost field-programmable gate array (FPGA) logic chip taking advantage of its high-speed serializers/deserializers (SerDes) [19]. Moreover, one FPGA chip can control many transmitters in parallel which results in a simpler design and overall system cost reduction. The control bits $a_{i}, i \in\left\{\log _{2} M_{c}, \log _{2} M_{c}+1, \ldots, \log _{2} M-1\right\}$ are used to control the relative optical delays between the EO switches, which are either 0 or $2^{i-\log _{2} M_{c}} \tau / \gamma$, depending on the value of $a_{i}$, while the control bits $a_{i}, i \in\left\{0,1, \ldots, \log _{2} M_{c}-1\right\}$ control which one of the $M_{c}$ OCDM encoders will be used. The maximum bit rate per user supported by the system is limited by the EO switches configuring latency (equal FPGA processing time and EO switch switching time). It is worthwhile noting that this latency should be $\leq T_{O P P M}$. One advantage of using CSK under same bit constraint is the increase of $T_{c}$ and consequently $T_{O P P M}$, hence eliminating the need for highswitching speeds. High-speed compact Mach-Zehnder-type EO switches, with switching times $<2.5 \mathrm{~ns}$, have been successfully fabricated [20]. A silicon EO switch, consisting of double-ring assisted Mach-Zehnder interferometer with $\mathrm{GHz}$ speed, has been demonstrated in [21]. In OCDM encoder, the frequency components of the optical pulse are selectively demultiplexed into $w$ wavelength components according to the wavelength hopping pattern in the corresponding signature code. Then each component is appropriately time-delayed using optical tapped delay lines (TDLs) in accordance with the time pattern of the 


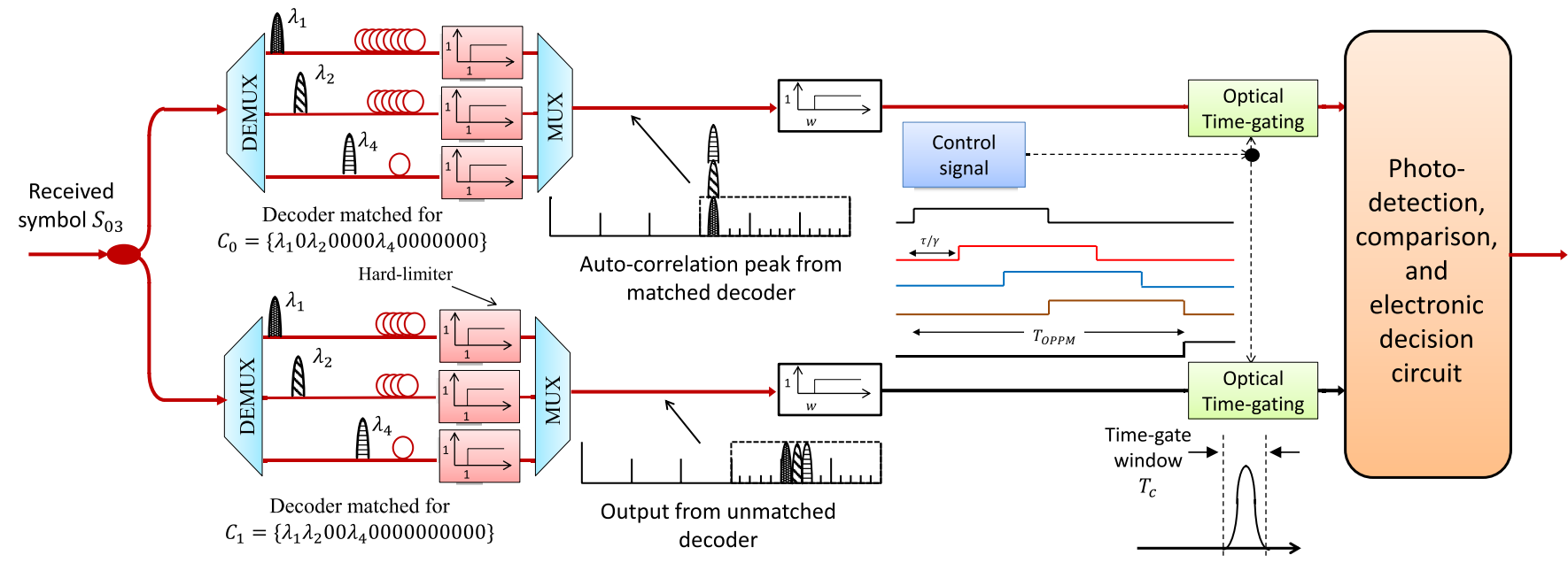

Fig. 2. CSK/OPPM-OCDM decoder for $M_{c}=2, M_{p}=4, \gamma=3, L=15$, and $w=3$.

signature code and then recombined using a multiplexer to generate the CSK/OPPM-OCDM signal. The CSK/OPPM-OCDM signal is then combined with signals from other transmitters and transmitted across the passive $N \times N$ star coupler to all receivers. Fig. 1 schematically shows the proposed CSK/OPPMOCDM transmitter for $M_{c}=2, M_{p}=4$ and $\gamma=3$, where bits 110 are mapped into slot $M_{p}-1$ and code $C_{0}$.

\section{B. Receiver Side}

At the receiver side, there are $M_{c}$ parallel OCDM decoders with double optical hard-limiters, each matched to one of the $M_{c}$ assigned codes to user $k$. Fig. 2 illustrates the schematic of the CSK/OPPM-OCDM receiver for user $k$ for $M_{c}=2$, $M_{p}=4$, and $\gamma=3$. In the OPPM demodulation after each OCDM decoder, a time-gate opens a time window of $T_{c}$ at the start of each $M_{p}$ slots constituting the OPPM frame. The gated pulses are then photodetected for decision making. The OPPM demodulator is merely a comparator that determines which slot in the $M_{p}$ time slots for which one of the $M_{c}$ codes has the largest photon count to be declared as the transmitted symbol.

Fig. 3 illustrates an example of the transmitted CSK/OPPMOCDM signal formats of a single user. In this figure two 2D OCFHC/OOC codes (i.e., $M_{c}=2$ ) and four overlapping positions (i.e., $M_{p}=4$ ) with overlapping index $\gamma=3$ are used to create 2-4 CSK/OPPM-OCDM. In the figure, $S_{i j}, i \in$ $\left\{0,1, \ldots, M_{c}-1\right\}, j \in\left\{0,1, \ldots, M_{p}-1\right\}$, represents the symbol $i j$ which use code $i$ within slot $j$.

\section{Performance Analysis}

In this section, we analyze the performance of the proposed system using APD. In our derivation, we assume a slot-asynchronous chip-synchronous system for ease of computation. In the more realistic case of chip-asynchronous, the performance will be superior to the case of chip-synchronous since the latter provides BEP upper bound [22]. We start by the BEP of traditional OOK-OCDM system, followed by the proposed CSK/OPPM-OCDM system.

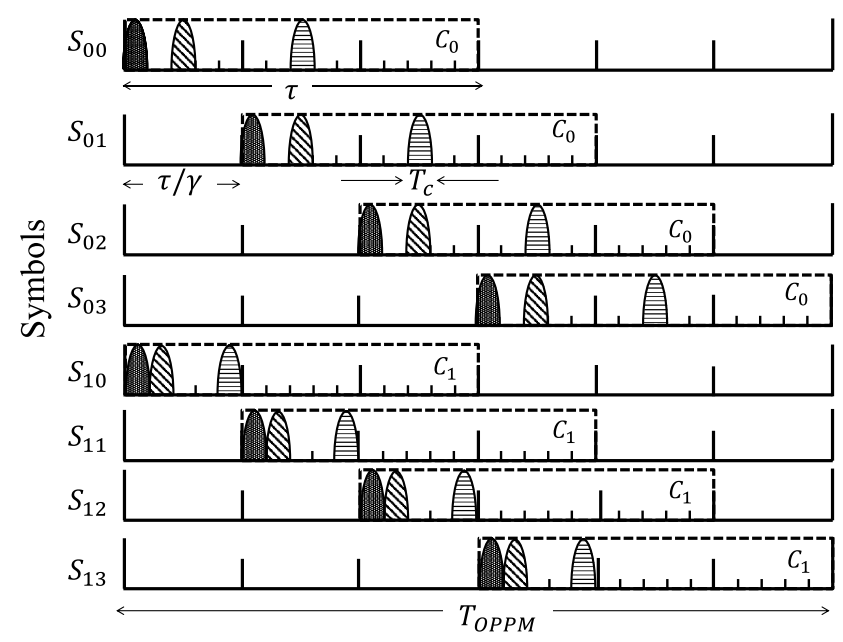

Fig. 3. CSK/OPPM-OCDM signaling scheme with $M_{C}=2$, $M_{p}=4, \quad \gamma=3 \quad L=15, \quad$ and $\quad w=3$. A codeset of $C_{i} \in$ $\left\{\left(\lambda_{1} 0 \lambda_{2} 0000 \lambda_{4} 0000000\right),\left(\lambda_{1} \lambda_{2} 00 \lambda_{4} 000000000\right)\right\}$ is assumed.

\section{A. BEP of OOK-OCDM System}

The correlation receiver with hard-limiters decides a data bit "1" was transmitted if the collected photon count in any bit interval is greater than a decision threshold $\theta$. A data bit " 0 " is decided otherwise. For large number of incident photons, the APD output can be approximated by a Gaussian random variable [23]. Let $Y$ denote the photon count collected in one bit interval, then the BEP can be evaluated as described in [24] as follows:

$$
\begin{aligned}
& P_{e}=\frac{1}{2}[\operatorname{Pr}\{Y \leq \theta \mid 1\}+\operatorname{Pr}\{Y>\theta \mid 0\}] \\
&=\frac{1}{2}\left[Q\left(\frac{\mu_{1}-\theta}{\sigma_{1}}\right)+Q\left(\frac{\theta-\mu_{1}}{\sigma_{1}}\right) \operatorname{Pr}\{Z=w\}\right. \\
&\left.+Q\left(\frac{\theta-\mu_{0}}{\sigma_{0}}\right) \operatorname{Pr}\{Z \neq w\}\right],
\end{aligned}
$$

where the factor $1 / 2$ is due to equiprobable data bits $(\operatorname{Pr}\{0\}=$ $\operatorname{Pr}\{1\}=1 / 2), Q(x) \stackrel{\text { def }}{=}(1 / \sqrt{2 \pi}) \int_{x}^{\infty} e^{-y^{2} / 2} d y$. Moreover, $\mu_{0 / 1}$ 
and $\sigma_{0 / 1}^{2}$ denote the mean and variance of the receiver output conditional on the transmitted bit being $0 / 1$, respectively. The random variable $Z \in\{0,1, \ldots, w\}$ denotes the number of interfered mark positions in the bit interval of the desired user immediately after the first optical hardlimiter. $\operatorname{Pr}\{Z=w\}$ is given as [25]:

$$
\begin{aligned}
\operatorname{Pr}\{Z=w\} & =\operatorname{Pr}\left\{\alpha_{1} \geq 1, \alpha_{2} \geq 1, \ldots, \alpha_{w} \geq 1\right\} \\
& =\sum_{t=0}^{w}(-1)^{t}\left(\begin{array}{c}
w \\
t
\end{array}\right)\left(1-t \frac{q}{w}\right)^{N-1},
\end{aligned}
$$

where $\alpha_{t}, t \in \chi \stackrel{\text { def }}{=}\{1,2, \ldots, w\}$ denotes the number of interference hits (or coincidences) occurred in the $t$ th marked chip of the desired user. Since $\sum_{z=0}^{w} \operatorname{Pr}\{Z=z\}=1$, then

$$
\operatorname{Pr}\{Z \neq w\}=1-\operatorname{Pr}\{Z=w\} .
$$

To minimize the BEP, the decision threshold $\theta$ is taken as $\theta=\left(\mu_{0} \sigma_{1}+\mu_{1} \sigma_{0}\right) /\left(\sigma_{0}+\sigma_{1}\right)[26]$, then

$$
P_{e}=\frac{1}{2} \operatorname{Pr}\{Z=w\}+Q\left(\frac{\mu_{1}-\mu_{0}}{\sigma_{1}+\sigma_{0}}\right) \operatorname{Pr}\{Z \neq w\} .
$$

The average interference hit probability for 2D OCFHC/OOC codes is given by [5]:

$$
q=\frac{\Phi_{C^{0}}}{\Phi_{C}} q^{0}+\frac{\Phi_{C^{1}}}{\Phi_{C}} q^{1},
$$

where $\Phi_{C^{0}}=p^{k} \Phi_{O O C}$ and $\Phi_{C^{1}}=p^{k}\left(1-p^{k}\right) \Phi_{O O C}$ denote the cardinality of codewords in group $C^{0}$ and $C^{1}$, respectively. $\Phi_{O O C}=\lfloor(L-1) / w(w-1)\rfloor$ represents the cardinality of $(L, w, 1,1)$ OOC used for TS. The overall cardinality $\Phi_{C}$ is given by:

$$
\Phi_{C}=\Phi_{C^{0}}+\Phi_{C^{1}}=m^{2} \Phi_{O o C},
$$

where $m=p^{k}$ represents the number of available wavelengths, $p$ is a prime number, and $k$ is the degree of the primitive polynomial $f(x)$ over Galois field $G F(p) . q^{0}$ and $q^{1}$ denote the one hit probabilities between the desired codeword originated from group $C^{0}$ or group $C^{1}$ and any interfering codeword in the code set, respectively. They are given by [5]:

$$
q^{0}=\frac{w^{2}\left(m \Phi_{O O C}-1\right)}{2 L\left(\Phi_{C}-1\right)}, \quad q^{1}=\frac{w^{2} m \Phi_{O O C}-w}{2 L\left(\Phi_{C}-1\right)},
$$

where the factor $1 / 2$ comes from the assumption of equiprobable on-off data-bit transmission, and $\Phi_{C}-1$ is the number of possible interferers. Substituting (7) in (5) we get $q=$ $w^{2} R / 2 m L$, where

$$
R=\frac{\left(\Phi_{C}-1\right)-(m-1) / w}{\left(\Phi_{C}-1\right)}
$$

represents the fraction of the number of interfering codewords contributing one hit (due to cross-correlation function) to the total number of interfering codewords (as there is some codewords in 2D OCFHC/OOC have zero cross-correlation).
The expressions for the parameters $\mu_{0} \sigma_{0}^{2} \mu_{1}$, and $\sigma_{1}^{2}$ for $2 \mathrm{D}$ OCFHC/OOC OCDM system are adapted here from [7], [27]:

$$
\begin{aligned}
\mu_{0}= & \frac{1}{2} \frac{\langle G\rangle \sum_{j=1}^{w} n_{j}}{k \lambda}\left(1-e^{-k \lambda}\right)+n_{d}\langle G\rangle \\
\sigma_{0}^{2}= & \frac{1}{4} \frac{\langle G\rangle^{2}\left(\sum_{j=1}^{w} n_{j}\right)^{2}}{k \lambda^{2}} \frac{\left(1-e^{-k \lambda}\right)^{4}}{\left(1-e^{-2 k \lambda}\right)} \\
& +\frac{\langle G\rangle^{2} F \sum_{j=1}^{w} n_{j}}{2 k \lambda}\left(1-e^{-k \lambda}-k \lambda e^{-k \lambda}\right) \\
& +\frac{n_{d}\langle G\rangle^{2} F}{k \lambda}\left(k \lambda-1+e^{-k \lambda}\right)+\sigma_{J}^{2} \\
\mu_{1}= & \mu_{0}+\frac{\langle G\rangle \sum_{j=1}^{w} n_{j}}{k \lambda}\left(k \lambda-1+e^{-k \lambda}\right) \\
\sigma_{1}^{2}= & \sigma_{0}^{2}+\frac{\langle G\rangle^{2} F \sum_{j=1}^{w} n_{j}}{k \lambda}\left(k \lambda-2+(2+k \lambda) e^{-k \lambda}\right),
\end{aligned}
$$

where brackets represent ensemble averaging, $G$ denotes the stochastic APD gain, $k=4 B_{\text {sneq }} / 2 \pi B_{3 \mathrm{~dB}}$ represents bandwidth correction factor, $B_{\text {sneq }}$ is shot-noise-equivalent bandwidth, $B_{3 \mathrm{~dB}}$ is $3-\mathrm{dB}$ bandwidth of the $\mathrm{APD}$, and $\lambda=2 \pi B_{3 \mathrm{~dB}} / R_{c}$ is the detector's relative speed, where $R_{c}=1 / T_{c}$ is the chip transmission rate. Here, $n_{j}$ is the average number of absorbed photons per received single-user chip at wavelength $\lambda_{j}, j \in$ $\{1,2, \ldots, m\}$, given by:

$$
n_{j}=\frac{\eta_{j} \lambda_{j} P_{p, j} T_{c}}{h C},
$$

where $P_{p, j}$ and $\eta_{j}$ denote the peak power of the received chip and the quantum efficiency at $\lambda_{j}$, respectively, $C=3 \times 10^{8} \mathrm{~m} / \mathrm{s}$ is the vacuum speed of light, and $h=6.626 \times 10^{-34} \mathrm{~J} . \mathrm{s}$ is Planck's constant. The average single-user received laser power $P_{\text {av }}$ for OOK-OCDM will be

$$
P_{\mathrm{av}}=\frac{\sum_{j=1}^{w} P_{p, j} T_{c}}{2 \tau},
$$

where $\tau=L T_{c}$ is the OOK bit duration, and the factor $1 / 2$ is multiplied due to data bit " $O$ " is not transmitted. $F$ is the APD excess noise factor, defined as $F \stackrel{\text { def }}{=}\left\langle G^{2}\right\rangle /\langle G\rangle^{2}=k_{\mathrm{eff}}\langle G\rangle+$ $\left(1-k_{\text {eff }}\right)[2-(1 /\langle G\rangle)]$, where $k_{\text {eff }}$ is the APD effective ionization ratio [28]. Also, $n_{d}$ is the average number of dark carriers generated per chip time interval $T_{c}$, given by $n_{d}=I_{d} T_{C} / e$, where $I_{d}$ is the APD dark current, and $e=1.6 \times 10^{-19} \mathrm{C}$ is the electron charge. Finally, $\sigma_{J}^{2}=2 k_{B} T_{n} T_{c} / e^{2} R_{L}$ represents the variance of thermal noise within a chip interval where, $k_{B}=$ $1.38 \times 10^{-23} \mathrm{~J} /{ }^{o} \mathrm{~K}$ is Boltzmann's constant, $T_{n}$ is the receiver noise temperature, and $R_{L}$ is the receiver load resistance.

Assuming an optical chip pulse with a Gaussian shape propagating in a linear dispersive medium. The pulse broadening factor due to the effect of GVD can be expressed as [29]:

$$
\sigma_{D, j}=\frac{\tau_{d}}{\tau_{0}}=\left[\left(1+\frac{C_{p} \beta_{2, j} d}{2 \tau_{0}^{2}}\right)^{2}+\left(\frac{\beta_{2, j} d}{2 \tau_{0}^{2}}\right)^{2}\right]^{1 / 2},
$$


where $C_{p}$ is the chirp parameter, $\tau_{0}=T_{c} /(2 \sqrt{2 \ln 2})$ is the RMS pulse width, $\tau_{d}$ is pulse power after propagating a certain distance $d$ along fiber, $\beta_{2, j}=-D \lambda_{j}^{2} / 2 \pi C$ is the GVD factor at $\lambda_{j}$, and $D$ is the dispersion parameter. The peak power of the received laser chip is given as [29], [30]:

$$
P_{p, j}=\frac{10^{\left(\frac{P_{c}(\mathrm{dBm})-\alpha(\mathrm{dB} / \mathrm{km}) d(\mathrm{~km})}{10}\right)} \times 10^{-3}}{\sigma_{D, j}},
$$

where $\alpha$ is the attenuation coefficient. It is worth to mention that $P_{c}$ is assumed to be the same for all wavelengths and OCDM signaling schemes used in this paper.

\section{B. BEP of CSK/OPPM-OCDM System}

We define a $M_{c} \times M_{p}$ matrix $\mathscr{Y}$ whose element $Y_{i j}, i \in \mathcal{M}_{c} \stackrel{\text { def }}{=}$ $\left\{0,1, \ldots, M_{c}-1\right\}, j \in \mathcal{M}_{p} \stackrel{\text { def }}{=}\left\{0,1, \ldots, M_{p}-1\right\}$, represents the photon count collected from slot $j$ at the receiver matched to codeword $i$ as:

$$
\mathscr{Y} \stackrel{\text { def }}{=}\left(\begin{array}{cccc}
Y_{00} & Y_{01} & \ldots & Y_{0\left(M_{p}-1\right)} \\
Y_{10} & Y_{11} & \ldots & Y_{1\left(M_{p}-1\right)} \\
\ldots & & \ldots & \\
Y_{\left(M_{c}-1\right) 0} & Y_{\left(M_{c}-1\right) 1} & \ldots & Y_{\left(M_{c}-1\right)\left(M_{p}-1\right)}
\end{array}\right) .
$$

Data symbol $i j$ is declared to be sent if $Y_{i j}>Y_{x y}$ for every $x \in$ $\mathcal{M}_{c}, y \in \mathcal{M}_{p}$, and $x y \neq i j$. An incorrect decision is otherwise declared. The probability of symbol error $P_{S}$ is given by:

$$
P_{S}=\sum_{i=0}^{M_{c}-1} \sum_{j=0}^{M_{p}-1} \operatorname{Pr}\{\text { error } \mid S=i j\} \operatorname{Pr}\{S=i j\},
$$

where $S$ denotes the transmitted data symbol. Assuming equally probable data symbols, i.e., $\operatorname{Pr}\{S=i j\}=1 / M$, then $\operatorname{Pr}\{$ error $\mid S=i j\}$ is independent of $i j$. The bit error probability $P_{b}$ can be obtained from $P_{S}$ using the formula $P_{b}=[M / 2(M-$ 1)] $P_{S}$ [31]. Without loss of generality, we assume that $S=00$ (i.e., code 0 in slot 0 ). Using a union bound, the upper bound on $P_{S}$ is expressed as:

$$
\begin{aligned}
P_{S}= & \operatorname{Pr}\left\{Y_{i j} \geq Y_{00}, \text { some } i j \neq 00 \mid S=00\right\} \\
\leq & \sum_{i=1}^{M_{c}-1} \operatorname{Pr}\left\{Y_{i 0} \geq Y_{00} \mid S=00\right\} \\
& +\sum_{i=0}^{M_{c}-1} \sum_{j=1}^{M_{p}-1} \operatorname{Pr}\left\{Y_{i j} \geq Y_{00} \mid S=00\right\} \\
= & \left(M_{c}-1\right) \operatorname{Pr}\left\{Y_{10} \geq Y_{00} \mid S=00\right\} \\
& +M_{c}\left(M_{p}-1\right) \operatorname{Pr}\left\{Y_{01} \geq Y_{00} \mid S=00\right\},
\end{aligned}
$$

where the first probability in the last equation is due to the photon count of the symbols that have same position with that of slot 00 (due to CSK effect). It can be evaluated as:

$$
\begin{aligned}
& \operatorname{Pr}\left\{Y_{10} \geq Y_{00} \mid S=00\right\} \\
& =\operatorname{Pr}\left\{Y_{10} \geq Y_{00} \mid S=00, Z_{10}=w\right\} \operatorname{Pr}\left\{Z_{10}=w\right\} \\
& \quad+\operatorname{Pr}\left\{Y_{10} \geq Y_{00} \mid S=00, Z_{10} \neq w\right\} \operatorname{Pr}\left\{Z_{10} \neq w\right\},
\end{aligned}
$$

where $Z_{i j} \in \chi, i \in \mathcal{M}_{c}, j \in \mathcal{M}_{p}$, is a random variable that denotes the number of interfered mark positions in slot $i j$ of the desired user immediately after the first optical hardlimiter. Let $\alpha_{t}^{10}, t \in \chi \stackrel{\text { def }}{=}\{1,2, \ldots, w\}$, denote the number of hits occurred in the $t$ th mark of undesired slot 10 . Then $\operatorname{Pr}\left\{Z_{10}=w\right\}$ is given by [25]:

$$
\begin{aligned}
\operatorname{Pr}\left\{Z_{10}=w\right\} & =\operatorname{Pr}\{w \text { marks interfered in solt } 10\} \\
& =\operatorname{Pr}\left\{\alpha_{1}^{10} \geq 1, \alpha_{2}^{10} \geq 1, \ldots, \alpha_{w}^{10} \geq 1\right\} \\
& =\sum_{t=0}^{w}(-1)^{t}\left(\begin{array}{c}
w \\
t
\end{array}\right)\left(1-t \frac{p_{X}}{w}\right)^{N-1},
\end{aligned}
$$

where $p_{X}$ is the probability of an external interference from other users in the network adopted for 2-D OCFHC/OOC OCDM system, given by [17]:

$$
p_{X}=\frac{w^{2} R}{\left(M_{p}-1+\gamma\right) m L} \gamma .
$$

Modeling the discrete outputs of APD, $Y_{00}$ and $Y_{10}$, as continuous Gaussian random variables [23], we get

$$
\begin{aligned}
& \operatorname{Pr}\left\{Y_{10} \geq Y_{00} \mid S=00, Z_{10}=w\right\} \\
& \quad \leq \operatorname{Pr}\left\{V=Y_{10}-Y_{00} \geq 0 \mid S=00, Z_{10}=w\right\} \\
& \quad=Q\left(-\frac{\mu_{v}}{\sigma_{v}}\right) \\
& \operatorname{Pr}\left\{Y_{10} \geq Y_{00} \mid S=00, Z_{10} \neq w\right\} \\
& \quad \leq \operatorname{Pr}\left\{U=Y_{10}-Y_{00} \geq 0 \mid S=00, Z_{10} \neq w\right\} \\
& \quad=Q\left(-\frac{\mu_{u}}{\sigma_{u}}\right),
\end{aligned}
$$

where $\mu_{v}$ and $\sigma_{v}^{2}$ denote the mean and variance of the random variable $V=Y_{10}-Y_{00}$ conditioned on $Z_{10}=w$. Also $\mu_{u}$ and $\sigma_{u}^{2}$ are the mean and variance of the random variable $U=$ $Y_{10}-Y_{00}$ conditioned on $Z_{10} \neq w$. The expressions for the parameters $\mu_{v}, \sigma_{v}^{2}, \mu_{u}$ and $\sigma_{u}^{2}$ are [24]:

$$
\begin{aligned}
\mu_{v} & =\mu_{1}-\mu_{1}=0, & \mu_{u} & =\mu_{0}-\mu_{1}, \\
\sigma_{v}^{2} & =2 \sigma_{1}^{2}, & \sigma_{u}^{2} & =\sigma_{0}^{2}+\sigma_{1}^{2},
\end{aligned}
$$

respectively. It is worth noting that in OPPM, $P_{\mathrm{av}}=$ $\sum_{j=1}^{w} P_{p, j} T_{c} / T_{\mathrm{OPPM}}$. The last probability in (16) is due to the 
photon count of the symbols that have different positions with that of slot 00 (OPPM effect), and it can be evaluated as:

$$
\begin{aligned}
\operatorname{Pr}\left\{Y_{01} \geq Y_{00} \mid S=00\right\} \\
=\operatorname{Pr}\left\{Y_{01} \geq Y_{00} \mid S=00, Z_{01}=w\right\} \operatorname{Pr}\left\{Z_{01}=w\right\} \\
\quad+\operatorname{Pr}\left\{Y_{01} \geq Y_{00} \mid S=00, Z_{01} \neq w\right\} \operatorname{Pr}\left\{Z_{01} \neq w\right\}(22) \\
\quad+\operatorname{Pr}\left\{Y_{01} \geq Y_{00}, v_{I}=1 \mid S=00, P_{2}\right\} \times \operatorname{Pr}\left\{P_{2}\right\} \\
\quad+\operatorname{Pr}\left\{Y_{01} \geq Y_{00}, v_{I}=1 \mid S=00, P_{3}\right\} \times \operatorname{Pr}\left\{P_{3}\right\},
\end{aligned}
$$

where $\operatorname{Pr}\left\{Z_{01}=w\right\}$ is given by (18) and we have the following further bound:

$$
\begin{aligned}
& \operatorname{Pr}\left\{Y_{01} \geq Y_{00} \mid S=00, Z_{01}=w\right\} \operatorname{Pr}\left\{Z_{01}=w\right\} \\
& +\operatorname{Pr}\left\{Y_{01} \geq Y_{00} \mid S=00, Z_{01} \neq w\right\} \operatorname{Pr}\left\{Z_{01} \neq w\right\} \\
& \quad \leq \frac{1}{2} \operatorname{Pr}\left\{Z_{01}=w\right\}+Q\left(-\frac{\mu_{u}}{\sigma_{u}}\right) \operatorname{Pr}\left\{Z_{01} \neq w\right\} .
\end{aligned}
$$

In the third term in (22), $P_{2}$ represents the event that $w-1$ marked chips from the $w$ marked chips in slot 01 are hit at least by one interfering pulse. Hence, $\operatorname{Pr}\left\{P_{2}\right\}$ is given by:

$$
\begin{aligned}
& \operatorname{Pr}\left\{P_{2}\right\}= \operatorname{Pr}\{w-1 \text { from } w \text { marks interfered in slot } 01\} \\
&=w \times \operatorname{Pr}\left\{\alpha_{1}^{01} \geq 1, \ldots, \alpha_{w-1}^{01} \geq 1, \alpha_{w}^{01}=0\right\} \\
&=w \times \quad \operatorname{Pr}\left\{\alpha_{1}^{01} \geq 1, \ldots, \alpha_{w-1}^{01} \geq 1\right\} \\
&\left.\quad-\operatorname{Pr}\left\{\alpha_{1}^{01} \geq 1, \ldots, \alpha_{w-1}^{01} \geq 1, \alpha_{w}^{01} \geq 0\right\}\right] \\
&=w \times\left[\sum_{t=0}^{w-1}(-1)^{t}\left(\begin{array}{c}
w-1 \\
t
\end{array}\right)\left(1-t \frac{q}{w}\right)^{N-1}\right. \\
&\left.\quad-\sum_{t=0}^{w}(-1)^{t}\left(\begin{array}{c}
w \\
t
\end{array}\right)\left(1-t \frac{q}{w}\right)^{N-1}\right] \\
&=w \times\left[\operatorname{Pr}\left\{Z_{01}^{\prime}=w-1\right\}-\operatorname{Pr}\left\{Z_{01}=w\right\}\right],
\end{aligned}
$$

where $Z_{01}^{\prime} \in\{0,1, \ldots, w-1\}$ is a random variable that denotes the number of interfered mark positions in slot 01 of the desired user immediately after the first optical hardlimiter. Also $P_{3}$ in (22) represents the event that the number of marked chips interfered at least by one interfering pulse not equal $w-1$, and it is given by:

$$
\begin{gathered}
\operatorname{Pr}\left\{P_{3}\right\}=\operatorname{Pr}\{\text { marks interfered in slot } 01 \neq w-1\} \\
=\sum_{\substack{i=0 \\
i \neq 1}}^{w}\left(\begin{array}{c}
w \\
i
\end{array}\right) \operatorname{Pr}\left\{\alpha_{1}^{01} \geq 1, \ldots, \alpha_{w-i}^{01} \geq 1,\right. \\
\left.\alpha_{w-i+1}^{01}=0, \ldots, \alpha_{w}^{01}=0\right\} \\
=1-\left(\begin{array}{c}
w \\
1
\end{array}\right) \operatorname{Pr}\left\{\alpha_{1}^{01} \geq 1, \ldots, \alpha_{w-1}^{01} \geq 1, \alpha_{w}^{01}=0\right\} \\
=1-w \times\left[\operatorname{Pr}\left\{Z_{01}^{\prime}=w-1\right\}-\operatorname{Pr}\left\{Z_{01}=w\right\}\right] .
\end{gathered}
$$

In (22), $v_{I} \in\{0,1\}$ is the number of pulses that cause self interference from slot 00 onto other undesired slots with different positions from that of symbol 00 . The average probability of self interference on a specific mark of an undesired slot is $p_{I} / w$.
TABLE I

SYSTEM PARAMETERS FOR NUMERICAL EVALUATIONS

\begin{tabular}{lll}
\hline \hline Name & Symbol & Value \\
\hline APD responsivity & $\Re=e \eta_{j} \lambda_{j} / h C$ & $0.84 \mathrm{~A} / \mathrm{W}$ \\
APD gain & $\langle G\rangle$ & 100 \\
APD effective ionization ratio & $k_{\text {eff }}$ & 0.02 \\
APD dark current & $I_{d}$ & $1 \mathrm{nA}$ \\
Wavelength range & $\lambda_{j}$ & $1550.0-1552.8 \mathrm{~nm}$ \\
Data bit rate & $R_{b}$ & $(0.4 \mathrm{~nm}$ separation $)$ \\
Chip duration & $T_{c}$ & $250 \mathrm{Mb} / \mathrm{s}$ \\
Receiver load resistance & $R_{L}$ & $0.02 \mathrm{~ns}$ \\
Receiver noise temperature & $T_{n}$ & $50 \Omega$ \\
Attenuation coefficient & $\alpha$ & $300,1000 \mathrm{~K}$ \\
Dispersion parameter & $D$ & $0.2 \mathrm{~dB} / \mathrm{km}$ \\
Chirp parameter & $C_{p}$ & $15 \mathrm{ps} / \mathrm{nm} / \mathrm{km}$ \\
\hline \hline
\end{tabular}

For the case $M_{p}-1+\gamma<2 \gamma+1, p_{I}$ for 2D OCFHC/OOC OCDM can be calculated as [18]:

$$
p_{I}=\sum_{\substack{i=-1 \\ i \neq 0}}^{\gamma-1} \frac{w^{2} R}{M_{p} m L}\left(1-\frac{|i|}{\gamma}\right)=\frac{w^{2} R}{M_{p} m L} \frac{\left(\gamma^{2}+\gamma-2\right)}{2 \gamma} .
$$

While for the case $M_{p}-1+\gamma \geq 2 \gamma+1, p_{I}$ is given as:

$$
p_{I}=\sum_{\substack{i=-(\gamma-1) \\ i \neq 0}}^{\gamma-1} \frac{w^{2} R}{M_{p} m L}\left(1-\frac{|i|}{\gamma}\right)=\frac{w^{2} R}{M_{p} m L}(\gamma-1) .
$$

Since $\left\{Y_{01} \geq Y_{00} \mid S=00\right\}$ and $\left\{v_{I}=1 \mid S=00\right\}$ are independent [32], we can write:

$$
\begin{aligned}
& \operatorname{Pr}\left\{Y_{01} \geq Y_{00}, v_{I}=1 \mid S=00, P_{2}\right\} \\
& \quad=\operatorname{Pr}\left\{v_{I}=1 \mid S=00, P_{2}\right\} \times \operatorname{Pr}\left\{Y_{01} \geq Y_{00} \mid S=00, P_{2}\right\} \\
& \quad=\frac{1}{w} p_{I} \times \operatorname{Pr}\left\{Y_{01} \geq Y_{00} \mid S=00, P_{2}\right\} \leq \frac{1}{2} \frac{1}{w} p_{I} .
\end{aligned}
$$

We can evaluate the fourth term in (22) in a similar way:

$$
\begin{aligned}
\operatorname{Pr} & \left\{Y_{01} \geq Y_{00}, v_{I}=1 \mid S=00, P_{3}\right\} \\
& =\frac{1}{w} p_{I} \times \operatorname{Pr}\left\{Y_{01} \geq Y_{00} \mid S=00, P_{3}\right\} \\
& \leq \frac{1}{w} p_{I} Q\left(-\frac{\mu_{u}}{\sigma_{u}}\right) .
\end{aligned}
$$

Finally, the symbol error rate is obtained as

$$
\begin{aligned}
P_{S} \leq & \left(M_{c}-1\right)\left[\frac{1}{2} \operatorname{Pr}\left\{Z_{10}=w\right\}+Q\left(-\frac{\mu_{u}}{\sigma_{u}}\right) \operatorname{Pr}\left\{Z_{10} \neq w\right\}\right] \\
& +M_{c}\left(M_{p}-1\right)\left[\frac{1}{2} \operatorname{Pr}\left\{Z_{01}=w\right\}+Q\left(-\frac{\mu_{u}}{\sigma_{u}}\right) \operatorname{Pr}\left\{Z_{01} \neq w\right\}\right. \\
& +\frac{1}{2} p_{I}\left(\operatorname{Pr}\left\{Z_{01}^{\prime}=w-1\right\}-\operatorname{Pr}\left\{Z_{01}=w\right\}\right)+Q\left(-\frac{\mu_{u}}{\sigma_{u}}\right) \\
& \left.\times \frac{1}{w} p_{I}\left[1-\left(\operatorname{Pr}\left\{Z_{01}^{\prime}=w-1\right\}-\operatorname{Pr}\left\{Z_{01}=w\right\}\right)\right]\right] .
\end{aligned}
$$

It is worth noticing that OPPM and CSK are special cases of CSK/OPPM when $M_{c}=1$ and $M_{p}=1$, respectively. Moreover, CSK/PPM is a special case of CSK/OPPM when $\gamma=1$. 


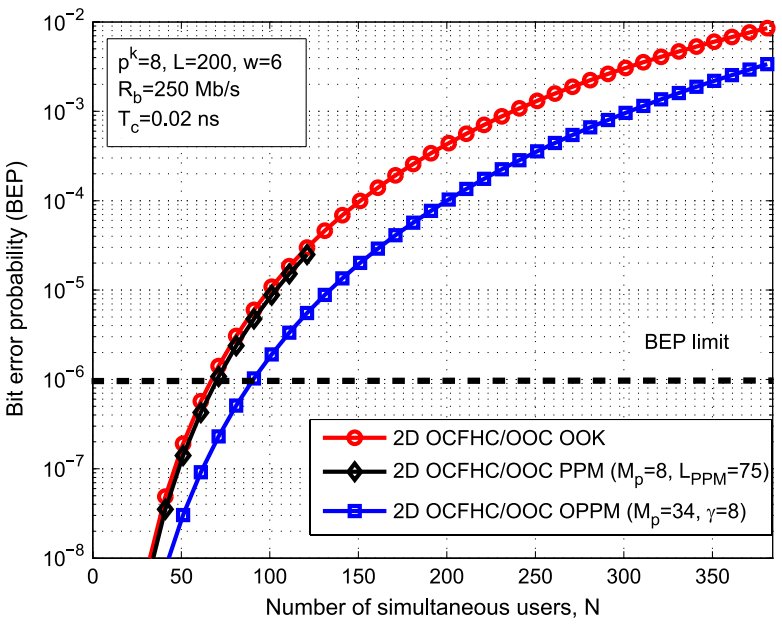

(a)

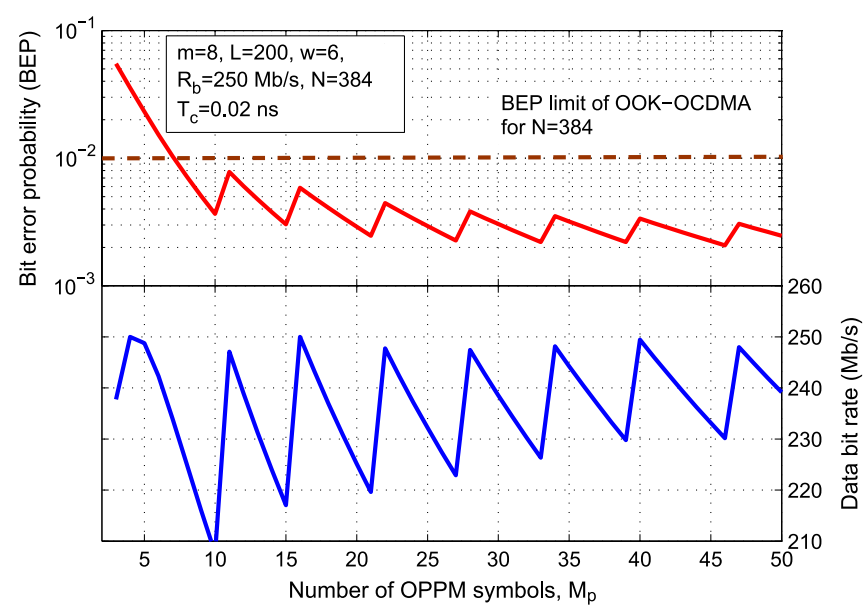

(b)

Fig. 4. (a) BEP of OOK-, PPM- and OPPM-OCDM systems versus the number of simultaneous users. (b) OPPM parameters selection. (Top) BEP; (Bottom) data bit rate fluctuations versus the number of OPPM symbols.

\section{NUMERICAL RESULTS}

The system parameters used in the analysis are provided in Table I. In our numerical calculations we have defined the target $\mathrm{BEP}$ to be $10^{-6}$. Moreover, we assume that the data rate per user $R_{b} \mathrm{~b} / \mathrm{s}$ is held fixed, and is given by:

$$
R_{b}=\frac{\log _{2} M}{T_{O P P M}}=\frac{\gamma \log _{2}\left(M_{c} \times M_{p}\right)}{\left(M_{p}-1+\gamma\right) L T_{c}}
$$

The BEP for both OOK-, PPM- and OPPM-OCDM systems for correlation receiver with hard-limiters considering only MAI is plotted in Fig. 4(a) against the number of simultaneous users $N$. We set $m=8, L=200$ and $w=6$ for OOK-OCDM system (i.e., $\Phi_{O O C}=\lfloor(L-1) / w(w-1)\rfloor=6$ and $\Phi_{C}=384$ ). In order to maintain the same $R_{b}$ using PPM system as in the OOK case with constraint on $T_{c}$, from (31) the code length for PPM system $L_{\mathrm{PPM}}$ will be $\left(L \log _{2} M_{p}\right) / M_{p}$. As a result the overall cardinality will be reduced as shown in Fig. 4(a). The OPPM parameters $M_{p}$ and $\gamma$ should be properly chosen as overlapping between slots may cause an increase in the error rate. The BEP is plotted for different values of $M_{p}$ in Fig. 4(b) (Top) with $N=384$ and $\gamma_{p}$ calculated using (31) such that $L, w, T_{c}$, and $R_{b}$ are the same with that of OOKOCDM. The proper values of $M_{p}$ and $\gamma$ are chosen such that the performance of OPPM system is better than that of OOK system. Fig. 4(b) (Bottom) shows the fluctuations in achieved $R_{b}$ because $\gamma_{p}$ should be integer. From Fig. 4(a), OOK-OCDM system can support 65 users $\left(\approx 17 \%\right.$ of $\left.\Phi_{C}=384\right)$ at BEP of $10^{-6}$, whereas OPPM-OCDM can support 90 users $(\approx 23 \%$ of $\left.\Phi_{C}=384\right)$. This is because OPPM-OCDM achieves higher tolerance to MAI, which results in improvement of BEP. From Fig. 4(a), the performance of PPM is nearly the same as OOK for $M_{p}=8$ case (it supports $\approx 67$ users at the target BEP of $10^{-6}$ ). For a much reduction of MAI, $M_{p}$ should be increased further. As a result, $L_{\mathrm{PPM}}$ will be reduced further limiting the number of available codewords.

Fig. 5 assess the effects of (i) APD noise and thermal noise, (ii) GVD, and (iii) device bandwidth and ISI on the OOK-

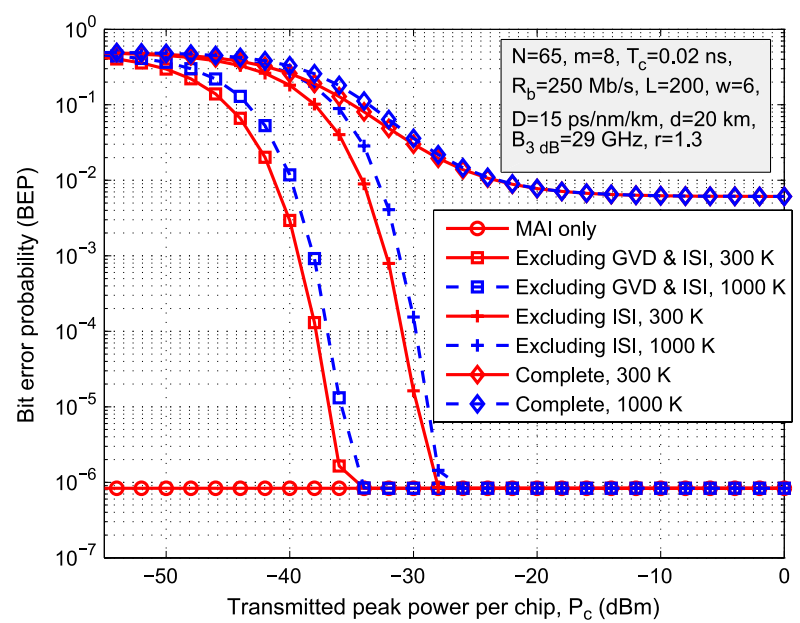

Fig. 5. BEPs versus transmitted power for OOK-OCDM system with different receiver temperatures.

OCDM system performance by plotting the BEP versus $P_{c}$ for the three different cases with $N=65$. Two different values of receiver noise temperatures $T_{n} \in\{300,1000\} \mathrm{K}$ are considered. The ratio $r=B_{\text {sneq }} / B_{3 \mathrm{~dB}}$ is assumed to be 1.3 , and $B_{3 \mathrm{~dB}}$ to be $29 \mathrm{GHz}$. GVD can be excluded from the calculations by setting $\beta_{2}=0$ in (12). APD bandwidth constraint and ISI was removed by assuming an instantaneous APD (i.e., $B_{3 \mathrm{~dB}}=\infty$ ). The effects of APD noise and thermal noise is predominant in the range of small transmitted power. However, when GVD effect is included, high transmitted power is required due to GVD power reduction. For the case of complete analysis (i.e., including APD noise, thermal noise, GVD, and ISI) the BEP of OOK-OCDM system greatly degraded because of the additional attenuation in the receiver output resulting from the APD's bandwidth constraint. Hence, the main factor that limits the system performance is ISI.

The corresponding curves for PPM-OCDM with $N=67$ and for OPPM-OCDM with $N=90$ systems are plotted in Fig. 6. For all three cases, PPM-OCDM has slightly better performance than that of OPPM-OCDM. This is because of 


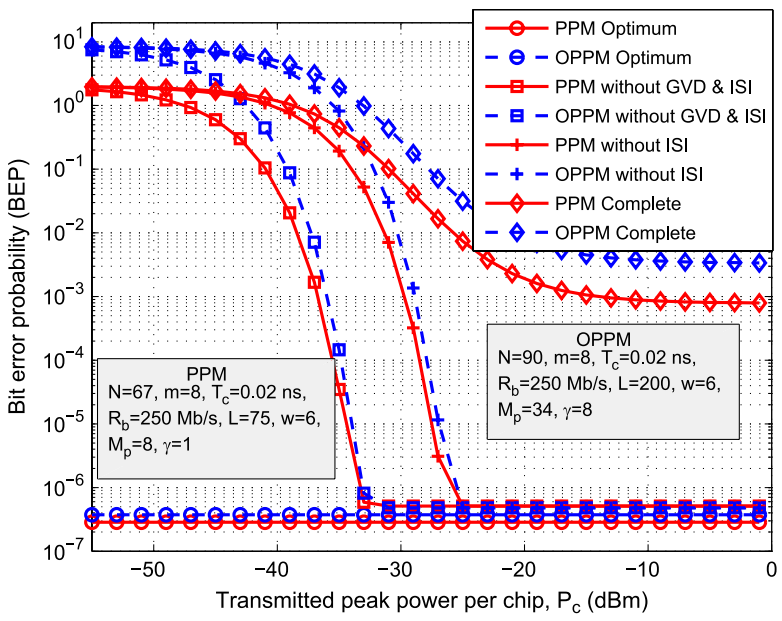

Fig. 6. BEPs versus transmitted power for PPM-OCDM and OPPM-OCDM systems.

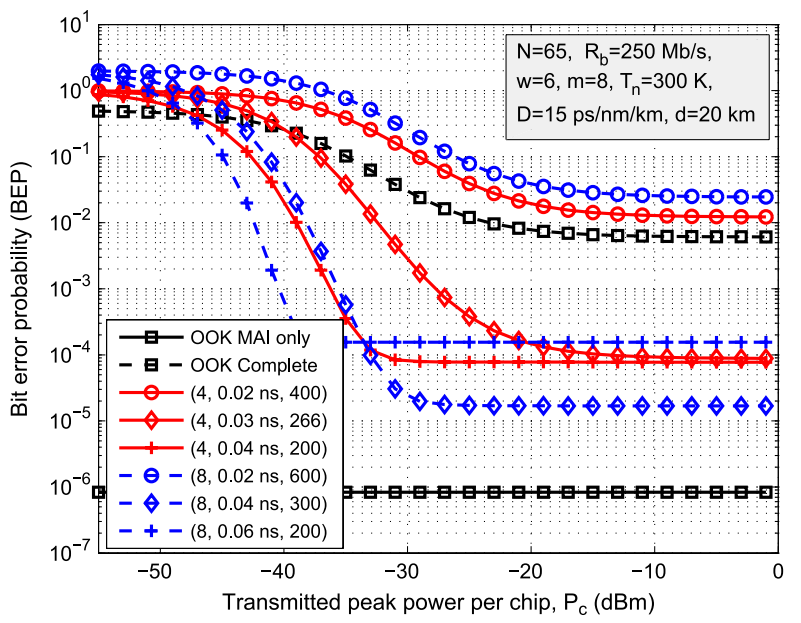

Fig. 7. BEPs versus transmitted power for CSK-OCDM.

overlapping between slots in OPPM which results in increasing the effects of MAI, APD noise, and thermal noise. However, it should be taking into consideration that these plots are at $N=67$ for PPM and $N=90$ for OPPM.

Fig. 7 compares the BEP of OOK and different cases of CSK (i.e., increasing the code length, increasing the chip duration, or both) versus the transmitted laser power per chip when $N=65$. In the figure, the numbers in the parentheses represent $M_{c} T_{c}$, and $L$, respectively. Increasing $L$ while keeping $T_{c}$ constant for the same transmission rate, the BEP of CSK gets worse because the added MAI from CSK. Increasing $T_{c}$ helps to reduce the chip transmission rate, hence relaxing the effects of both GVD and ISI. Simultaneously increasing $L$ and $T_{c}$ can reduce the effects of MAI, GVD, and ISI.

Fig. 8 shows the BEP for CSK/OPPM-OCDM system versus $P_{c}$ when $N=90$ for the different cases (i.e., increasing $L, T_{c}$, or both). Increasing $L$ only degrades the system performance due to the added MAI from CSK effect. With constraint on $R_{b} L$, and $\gamma$ and using $M_{c}=2$, the increase in $T_{c}$ is small (from $0.02 \mathrm{~ns}$ to $0.0237 \mathrm{~ns}$ ), hence the improvement in BEP is little. With constraints on both $R_{b}$ and $L, T_{c}$ can be increased largely by increasing $\gamma$, thus greatly reducing the effects of GVD and ISI (case $M_{c}=4, L=200$, and $T_{c}=0.04 \mathrm{~ns}$ which corresponds

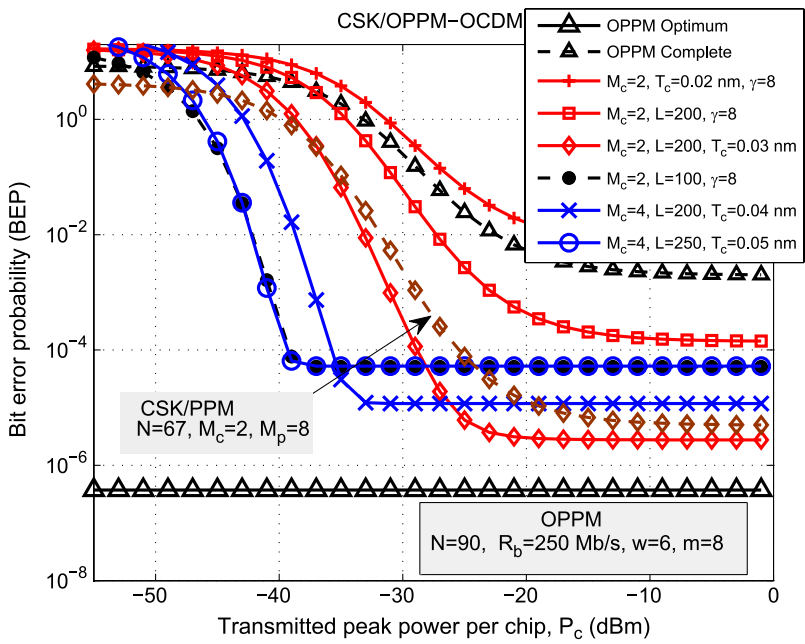

Fig. 8. BEPs versus transmitted power for CSK/OPPM-OCDM system.

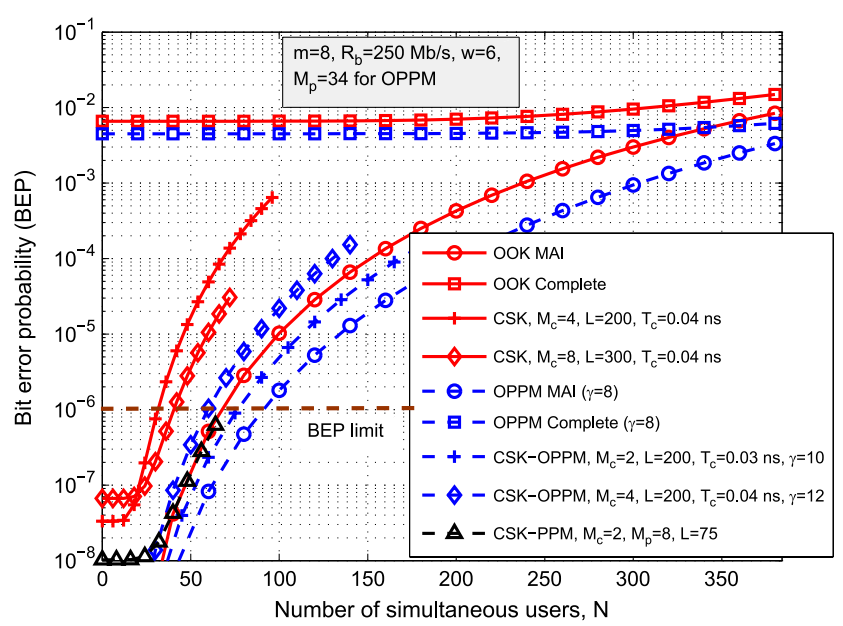

Fig. 9. BEPs versus the number of simultaneous users when $P_{c}=-15 \mathrm{dBm}$ and $d=20 \mathrm{~km}$.

to $\gamma=12$ ). However, increasing $T_{c}$ by further increasing $\gamma$ (while $M_{p}$ is fixed) will increase the effect of MAI on BEP which may be worse than GVD effect (case $M_{c}=4, L=250$, and $T_{c}=0.05 \mathrm{~ns}$ which corresponds to $\gamma=25$ ). This happens because the value of $M_{p}(=34)$ is not optimized for this large value of overlapping index. In order to overcome the effect of overlapping the parameters should be reoptimized to meet the new requirements. Due to the limited cardinality, only the case of $M_{c}=2, M_{p}=8$ and $N=67$ for CSK/PPM-OCDM is included in the figure as well.

Fig. 9 shows the BEP versus the number of simultaneous users with $P_{c}=-15 \mathrm{dBm}$ for CSK- and CSK/OPPM-OCDM systems. Comparing the MAI ideal cases (i.e., OOK MAI and OPPM MAI $(\gamma=8))$ with complete analysis cases (i.e., OOK Complete, and OPPM Complete), the performance degradation in the complete analysis cases due to ISI is even more severe for both OOK- and OPPM-OCDM systems and none of them can achieve the target BEP level of $10^{-6}$ (from figure the BEP $>10^{-3}$ ). Therefore, there is a need to use CSK- or CSK/OPPMOCDM to enhance the system performance as shown by the remaining curves in Fig. 9. Moreover, CSK/PPM case is also included in Fig. 9. However, the use of CSK-OCDM imposes more system complexity than OOK-OCDM system as multiple 
TABLE II

SPECTRAL EFFICIENCY FOR DIFFERENT OCDM SIGNALING SCHEMES

\begin{tabular}{|c|c|c|c|c|c|c|c|c|}
\hline $\begin{array}{l}\text { Signaling } \\
\text { scheme }\end{array}$ & $\begin{array}{l}\text { OOK } \\
\text { MAI }\end{array}$ & $\begin{array}{l}\text { CSK } \\
M_{c}=4 \\
T_{c}=0.04\end{array}$ & $\begin{array}{l}\text { CSK } \\
M_{c}=8 \\
T_{c}=0.04\end{array}$ & $\begin{array}{l}\text { OPPM } \\
\text { MAI }\end{array}$ & $\begin{array}{l}\text { CSK/OPPM } \\
M_{c}=2 \\
T_{c}=0.03\end{array}$ & $\begin{array}{l}\text { CSK/OPPM } \\
M_{c}=4 \\
T_{c}=0.04\end{array}$ & $\begin{array}{l}\text { PPM } \\
M_{p}=8 \\
T_{c}=0.02\end{array}$ & $\begin{array}{l}\text { CSK/PPM } \\
M_{c}=2, M_{p}=8 \\
T_{c}=0.026667\end{array}$ \\
\hline $\begin{array}{l}\text { Spectral } \\
\text { efficiency (\%) }\end{array}$ & $4.0625 \%$ & $3.75 \%$ & $5 \%$ & $5.625 \%$ & $7.2188 \%$ & $7.125 \%$ & $4.1875 \%$ & $5.3334 \%$ \\
\hline
\end{tabular}

OCDM encoders/decoders are needed. Moreover, the complexity increases when using CSK/OPPM-OCDM. The use of CSKOCDM provides a good compromise among high complexity of CSK/OPPM-OCDM and worse performance of OOK-OCDM and OPPM-OCDM systems.

Using the definition of spectral efficiency $\eta=K R_{b}$ / $m \Delta f,(\mathrm{~b} / \mathrm{s} / \mathrm{Hz})$, where $\Delta f=1 / T_{c}$ is the minimum required bandwidth [33] and $K$ is the number of supported users at the target BEP, the achieved spectral efficiency for the different signaling schemes is presented in Table II at BEP of $10^{-6}$ with the help of Fig. 9. From the figure, the proposed CSK/OPPMOCDM system achieves higher spectral efficiency than other systems.

\section{CONCLUSION}

An OPPM-OCDM system using CSK signaling has been proposed and theoretically investigated. Upper bound on the BEP for the proposed system has been obtained for correlation receiver with double-hard-limiters. The analysis includes the effects of MAI, GVD, APD noise, APD dark current, and thermal noise. Moreover, the effect of ISI has been also taken into account. Our numerical results indicate that ISI is the main performance limiting factor for OOK- and OPPM-OCDM systems. The proposed CSK/OPPM-OCDM system can mitigate the impacts of GVD, ISI, and MAI, consequently improving the overall performance of the network as well as achieving higher spectral efficiency when compared to traditional OOK-, PPM-, and OPPM-OCDM systems.

\section{REFERENCES}

[1] G. C. Yang and W. C. Kwong, "Performance comparison of the multiwavelength CDMA and WDMA+CDMA for fiber-optic networks," IEEE. Trans. Commun., vol. 45, no. 11, pp. 1426-1434, Nov. 1997.

[2] L. Tancevski, I. Andonovic, M. Tur, and J. Budin, "Hybrid wavelength hopping/time spreading code division multiple access systems," Proc. Inst Elect. Eng.—Optoelectron., vol. 143, no. 3, pp. 161-166, Jun. 1996.

[3] L. Tancevski and I. Andonovic, "Hybrid wavelength hopping/time spreading schemes for use in massive optical networks with increased security," J. Lightw. Technol., vol. 14, no. 12, pp. 2636-2646, Dec. 1996.

[4] W. C. Kong, G. C. Yang, V. Baby, C.-S. Bres, and P. R. Prucnal, "Multiplewavelength optical orthogonal codes under prime-sequence permutations for optical CDMA," IEEE Trans. Commun., vol. 53, no. 1, pp. 117-123, Jan. 2005.

[5] S. Shurong, H. Yin, Z. Wang, and A. Xu, "A new family of 2-D optical orthogonal codes and analysis of its performance in optical CDMA access networks," J. Lightw. Technol., vol. 24, no. 4, pp. 1646-1653, Apr. 2006.

[6] G. El-Howayek et al., "On the use of Gaussian approximation in analyzing the performance of optical receivers," IEEE Photon. J., vol. 6, no. 1, Feb. 2014, Art. ID. 6800508.

[7] D. S. G. Ong, J. S. Ng, M. M. Hayat, P. Sun, and J. P. R. David, "Optimization of InP APDs for high-speed lightwave systems," J. Lightw. Technol., vol. 27, no. 15, pp. 3294-3302, Aug. 2009.

[8] E. K. H. Ng, G. E. Weichenberg, and E. H. Sargent, "Dispersion in multiwavelength optical code division multiple-access systems: Impact and Remedies," IEEE Trans. Commun., vol. 50, no. 11, pp.1811-1816, Nov. 2002.

[9] S. P. Majumder, A. Azhari, and E. H. Hossam, "The impact of fiber chromatic dispersion on the BER performance of an optical CDMA IM/DD transmission system," IEEE Photon. Technol. Lett., vol.17, no. 6, pp.1340-1342, Jun. 2005.

[10] E. Narimanov, W. C. Kwong, G.-C. Yang, and P. R. Prucnal, "Shifted carrier-hopping prime codes for multicode keying in wavelength-time O-CDMA," IEEE Trans. Commun., vol. 53, no. 12, pp. 2150-2156, Dec. 2005.

[11] S. Galli, R. Menendez, and R. J. Runser, "A novel method for increasing the spectral efficiency of optical CDMA," IEEE Trans. Commun., vol. 56, no. 12, pp. 2133-2144, Dec. 2008.

[12] T. H. Shake, "Security performance of optical CDMA against eavesdropping," J. Lightw. Technol., vol. 23, no. 2, pp. 655-670, Feb. 2005.

[13] N. T. Dang and A. T. Pham, "Reducing the dispersion effects in multiwavelength optical CDMA systems by using MCM signaling," J. Opt. Commun. Netw., vol. 2, no. 11, pp. 967-974, Nov. 2010.

[14] E. D. J. Smith, R. J. Blaikie, and D. P. Taylor, "Performance enhancement of spectral-amplitude-coding optical CDMA using pulse-position modulation," IEEE Trans. Commun., vol. 46, no. 9, pp. 1176-1185, Sep. 1998.

[15] N. T. Dang, H. T. T. Pham, and A. T. Pham, "Novel multi-code pulse position modulation for performance improvement of 2-D OCDMA systems," in Proc. 17th APCC, Kota Kinabalu, Sabah, Malaysia, Oct. 2-5, 2011, pp. $96-101$.

[16] H. M. H. Shalaby, "Maximum achievable throughputs for uncoded OPPM and MPPM in optical direct-detection channels," J. Lightw. Technol., vol. 13, no. 11, pp. 2121-2128, Nov. 1995.

[17] H. M. H. Shalaby, "Direct detection optical overlapping PPM-CDMA communication systems with double optical hardlimiters," J. Lightw. Technol., vol. 17, no. 7, pp. 1158-1165, Jul. 1999.

[18] A. E. Farghal, H. M. H. Shalaby, and Z. Kawasaki, "Performance analysis of multirate multiservice optical CDMA networks adopting overlapping PPM signaling," J. Lightw. Technol., vol. 32, no. 15, pp. 2649-2658, Aug. 2014.

[19] Support: Virtex-7 Family Overview, Xilinx, San Jose, CA, USA, 2015 [Online]. Available: http://www.xilinx.com/support/documentation/data _sheets/ds180_7Series_Overview.pdf

[20] H. Kouketsu, S. Kawasaki, N. Koyama, Y. Matsushima, and K. Utaka, "High-speed and compact non-blocking $8 \times 8$ InAlGaAs/InAlAs MachZehnder-type optical switch fabric," presented at the Proc. Optical Fiber Communication Conf., San Francisco, CA, USA, 2014, Paper M2K.3.

[21] L. Lu, L. Zhou, X. Li, and J. Chen, "Low-power $2 \times 2$ silicon electro-optic switches based on double-ring assisted Mach-Zehnder interferometers," Opt. Lett., vol. 39, no. 6, pp. 1633-1636, Mar. 2014.

[22] T.-C. Wang, G.-C. Yang, C.-Y. Chang, and W. C. Kwong, "A new family of 2-D codes for fiber-optic CDMA systems with and without the chip-synchronous assumption," J. Lightw. Technol., vol. 27, no. 14, pp. 2612-2620, Jul. 2009.

[23] A. Papoulis, "High density shot noise and Gaussianity," J. Appl. Probability, vol. 8, no. 1, pp. 118-127, Mar. 1971.

[24] H. M. H. Shalaby, "Effect of thermal noise and APD noise on the performance of OPPM-CDMA receivers," J. Lightw Technol., vol. 18, no. 7, pp. 905-914, Jul. 2000

[25] S. Mashhadi and J. A. Salehi, "Code division multiple-access techniques in optical fiber networks-Part III: Optical AND gate receiver structure with generalized optical orthogonal codes," IEEE Trans. Commun., vol. 54, no. 8, pp. 1457-1468, Aug. 2006.

[26] G. P. Agrawal, Fiber-Optic Communication Systems, 3rd ed. New York, NY, USA: Wiley, 2002, p. 164

[27] P. Sun, M. M. Hayat, B. E. A. Saleh, and M. C. Teich, "Statistical correlation of gain and buildup time in APD and its effects on receiver performance," J. Lightw. Technol., vol. 24, no. 2, pp. 755-768, Feb. 2006.

[28] J. C. Campbell, "Recent advances in telecommunications avalanche photodiodes," J. Lightw. Technol., vol. 25, no. 1, pp. 109-121, Jan. 2007. 
[29] G. P. Agrawal, Nonlinear Fiber Optics, 3rd ed. New York, NY, USA: Academic, ch. 3, 2001.

[30] A. L. Sanches, J. V. dos Reis, Jr., and B.-H. V. Borges, "Analysis of highspeed optical wavelength/time CDMA networks using pulse-position modulation and forward error correction techniques," J. Lightw. Technol., vol. 27, no. 22, pp. 5134-5144, Nov. 2009.

[31] R. M. Gagliardi and S. Karp, Optical Communications, 2nd ed. New York, NY, USA: Wiley, 1995.

[32] A. G. Sabbagh and M. M. Kakhki, "Capacity analysis of asynchronous optical CDMA systems utilizing wrapped OPPM: Multiple access interference limited case," J. Lightw. Technol., vol. 31, no. 15, pp. 2613-2620, Aug. 2013.

[33] G. Manzacca, X. Wang, N. Wada, G. Cincotti, and K. Kitayama, "Comparative study of multiencoding schemes for OCDM using a single multiport optical encoder/decoder," IEEE Photon. Technol. Lett., vol. 19, no. 8, pp. 559-561, Apr. 2007.

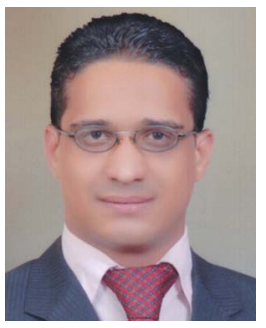

Ahmed E. Farghal received the B.S. (Hons.) and M.S. degrees from Menufiya University, Menouf, Egypt, in 2006 and 2011, respectively, and the Ph.D degree from Egypt-Japan University of Science and Technology (E-JUST), Alexandria, Egypt, in 2015 , all in electrical engineering. In 2007, he joined the Department of Electronics and Electrical Communications Engineering, Menufiya University, where he became a Lecturer Assistant in 2011. In November 2014 through July 2015, he was a Special Research Student with Kyushu University. He is currently with E-JUST. His research interests include fiber optic communication networks, optical code-division multiplexing, all-optical networks, elastic optical networks, and nano-optoelectronic devices.

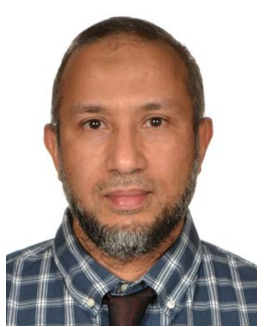

Hossam M. H. Shalaby (S'83-M'91-SM'99) was born in Giza, Egypt, in 1961. He received the B.S. and M.S. degrees from Alexandria University, Alexandria, Egypt, in 1983 and 1986, respectively, and the Ph.D. degree from the University of Maryland, College Park, MD, USA, in 1991, all in electrical engineering.

In 1991, he joined the Department of Electrical Engineering, Alexandria University, where he became a Professor in 2001. He is currently on leave from Alexandria University and is the Chair of the Department of Electronics and Communications Engineering, School of Electronics, Communications, and Computer Engineering, Egypt-Japan University of Science and Technology, Alexandria. From September 1996 to January 1998, he was with the Department of Electrical and Computer Engineering, International Islamic University of Malaysia, and from February 1998 to February 2001, he was with the School of Electrical and Electronic Engineering, Nanyang Technological University, Singapore. From September 1996 to February 2001, he was on leave from Alexandria University. From December 2000 to 2004, he was an Adjunct Professor with the Faculty of Sciences and Engineering, Department of Electrical and Information Engineering, Laval University, Quebec, QC, Canada. From 2007 to 2010, he was a Consultant at SysDSoft Company, Alexandria. His research interests include optical communications, silicon photonics, optical space-division multiplexing, optical CDMA, optical OFDM technology, and information theory.

Prof. Shalaby is a Senior member of the IEEE Photonics Society and The Optical Society (OSA). He served as a Student Branch Counselor at Alexandria University, IEEE Alexandria and North Delta Subsection, from 2002 to 2006, and as a Chairman of the Student Activities Committee of the IEEE Alexandria Subsection from 1995 to 1996 . He was a recipient of an SRC Fellowship from 1987 to 1991 from Systems Research Center, USA; the State Incentive Award in Engineering Sciences in 1995 and 2001 from the Academy of Scientific Research and Technology, Egypt; the University Incentive Award in 1996 from Alexandria University; the Shoman Prize for Young Arab Researchers in 2002 from the Abdul Hameed Shoman Foundation, Amman, Jordan; the State Excellence Award in Engineering Sciences in 2007 from the Academy of Scientific Research and Technology, Egypt; and the University Excellence Award in 2009 from Alexandria University.

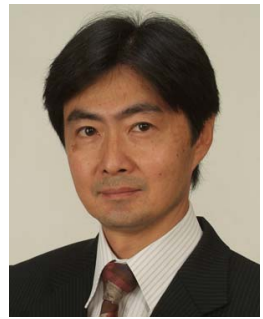

Kazutoshi Kato received the B.S. and M.S. degrees in physics and the Ph.D. degree from Waseda University, Tokyo, Japan, in 1985, 1987, and 1993, respectively. In 1987, he was with NTT Opto-Electronics Laboratories, Atsugi, Japan, where he was engaged in research on receiver OEICs, high-speed $\mathrm{p}-\mathrm{i}-\mathrm{n}$ PDs, and photoreceivers for wideband transmissions, microwave applications, and optical access networks. From 1994 to 1995, he was on leave from NTT at France Telecom CNET Bagneux Laboratory, Bagneux, France, as a Visiting Researcher working on MSM PDs. From 2000 to 2003, he was with NTT Electronics Corporation, where he was involved in developing photonic network systems. From 2009 to 2011, he was an Executive Manager at NTT Photonics Laboratories, Atsugi. $\mathrm{He}$ is currently a Professor of information science and electrical engineering with Kyushu University, Fukuoka, Japan. His current research interests include the advanced optoelectronics devices and subsystems for high-speed optical transmissions.

Dr. Kato is a Senior Member of the Institute of Electronics, Information and Communication Engineers (Japan) and the IEEE Photonics Society and a Member of the Japan Society of Applied Physics.

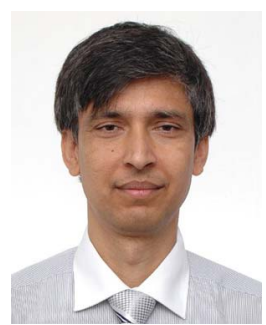

Ramesh K. Pokharel (M'04) received the M.E and Doctorate degrees in electrical engineering from The University of Tokyo, Tokyo, Japan, in 2000 and 2003, respectively. He had short academic and industrial experiences in Nepal before he joined The University of Tokyo in 1997 as a Research Student. From April 2003 to March 2005, he was a Postdoctoral Research Fellow with the Department of Electrical Engineering and Electronics, Aoyama Gakuin University, Tokyo. In April 2005, he joined the Department of Electronics, Graduate School of Information Science and Electrical Engineering, Kyushu University, where he is currently a Professor. From 2012 to 2013, he was the Vice Director of the Center for Egypt-Japan Cooperation in Science and Technology at Kyushu University, where he has been serving as the Director since April 2014. His current research interests include the low-cost RFIC and analog circuits for microwave and millimeter-wave wireless communications, on-chip signal integrity issues, and on-chip metamaterials in CMOS. Dr. Pokharel served as the Secretory of IEEE MTT-S Japan Society from January 2012 to December 2013 and has been serving as the Vice Chair of the Education Committee of the IEEE-MTT-S Japan Society since January 2014. He was a recipient of the Monbukagakusho Scholarship of the Japanese Government from 1997 to 2003 and an Excellent COE Research Presentation Award from the University of Tokyo in 2003. 